Te mátic a

\title{
Migrantes tZOTZILES EN LA VERTIENTE DEL MEZCALAPA Y EL CORAZÓN ZOQUE DE CHIAPAS, 1890-1940
}

\author{
María del Rocío Ortiz Herrera
}

Resumen: La migración temporal o definitiva que realizaron indígenas tzotziles de los Altos de Chiapas a las regiones del Soconusco y Motozintla, a finales del siglo XIX y las primeras décadas del XX, para incorporarse al trabajo en plantaciones $y$ fincas cafetaleras, ha sido objeto de numerosos trabajos. Pocos esfuerzos se han hecho para comprender la migración que en esos mismos años emprendieron familias tzotziles a otras regiones del estado, en las que también desempeñaron un papel importante como mano de obra o bien como pequeños productores que cultivaron tierras baldías o nacionales. Este texto pretende aportar elementos para comprender las otras rutas de la migración tzotzil de finales del siglo XIX y principios del $\chi X$, en particular la que realizaron algunas familias originarias de San Juan Chamula a los pueblos de Tapilula y Coapilla, región históricamente zoque.

Palabras clave: historia, migrantes, tzotziles, región zoque.

Enviado a dictamen: 06 de agosto de 2007. Aprobación: 10 de septiembre de 2007.

María del Rocío Ortiz Herrera, doctora en Ciencias Sociales, profesora investigadora de la licenciatura en Historia-CESMECA-UNICACH, temas de especialización: Iglesia, pueblos indios, historia agraria y procesos de pérdida de las lenguas indígenas, correo electrónico: ortizz7@yahoo.com.mx.
Abstract: The temporary or definitive migration of the Tzotzil Indians of the Highlands of Chiapas to the regions of the Soconusco and Motozintla, at the end of the $19^{\text {th }}$ century and the first decades of the $20^{\text {th }}$, in order to incorporate themselves in work on plantation and coffee farms, has been the object of many studies. Little effort has been made to understand the migrations that Tztotzil families, during the same years, who went to other regions of the state where they also played an important part as laborers, or as small producers who established themselves in empty or national lands. This text pretends to contribute additional information in order to understand the other routes of Tzotzil migration, during the last of the $19^{\text {th }}$ and beginnings of the $20^{\text {th }}$ centuries, especially those that Indian families from San Juan Chamula realized to the towns of Tapilula and Coapilla, a region historically zoque.

Key Words: History, migration, tzotzils, zoque region.

\section{Introducción}

T a reconstrucción histórica de los procesos migratorios supone el análisis de múltiples y complejos factores relacionados tanto con variables macrosociales - los contextos económicos, sociales y políticos más amplios-, como con realidades microsociológicas, es decir, con las actitudes, ideas, valores y percepción de los migrantes respecto de las presiones que experimentan sobre su vida social y económica. 
Estas variables microsociológicas, que son indispensables para comprender por qué los individuos de una determinada comunidad deciden migrar y otros no a pesar de compartir las mismas condiciones socioeconómicas, resultan casi siempre una tarea compleja para el historiador, pues las fuentes documentales difícilmente reflejan las ideas y valoraciones personales de quienes deciden abandonar temporal o de forma definitiva su lugar de origen. Los documentos históricos, en cambio, permiten reconstruir con mayor facilidad los factores macrosociales que intervienen en los procesos migratorios, aunque esos factores constituyen solamente una parte de la explicación.

En este texto analizo algunas de las variables económicas, demográficas y sociales más amplias que influyeron en la migración de indígenas tztotziles de los Altos de Chiapas a dos municipios de la región históricamente zoque, Tapilula y Coapilla, hacia finales del siglo XIX y los primeros años del XX. También busco dar cuenta de la situación que enfrentaron los migrantes durante los años de la Revolución y del reparto agrario en Chiapas una vez establecidos en esos pueblos.

Las fuentes que se consultaron consisten en periódicos oficiales, correspondencia de autoridades locales y estatales, además de anuarios y datos estadísticos. En el caso de Coapilla, diversos documentos de la década de 1,900 hacen referencia a indígenas tzotziles, originarios de San Juan Chamula, que se encontraban laborando como mozos o peones endeudados en una de las fincas más importantes del pueblo. Antes de establecerse en Coapilla, las familias migrantes habían radicado algún tiempo en Bochil, pero cuando el cultivo de café comenzó a cobrar importancia en Coapilla, hacia finales del siglo XIX, se incorporaron al trabajo de varias fincas.

Con respecto a Tapilula, en los documentos se menciona un núcleo de población de indígenas tzotziles, también originarios de San Juan Chamula, que cultivaban terrenos "baldíos" del municipio desde el año de 1916. Antes de esos años, las fuentes de archivo no registran la presencia de indígenas en ambos municipios, por lo que puede suponerse que la migración de los tzotziles a esos lugares se produjo a finales del siglo XIX o durante los primeros años del siglo XX.

\section{La región de partida: los Altos de Chiapas a finales del siglo XIX y principios del XX}

Los Altos de Chiapas — que en un sentido amplio abarca todos los municipios localizados en la franja meridional del macizo central, desde Zinacantán, San Cristóbal, Teopisca y Amatenango, en el sur, hasta los límites de ese conjunto montañoso con Tabasco, en el norte- era en el siglo XIX, como en la actualidad, la zona más poblada del estado y en donde habitaba el grueso de la población indígena. Esta circunstancia y el hecho de que una parte importante de las tierras de la región, sobre todo las que se localizan al sur, son terrenos poco propicios para la agricultura, definieron desde entonces la principal vocación de la zona: suministrar de mano de obra barata a otras regiones del estado poco pobladas, pero en donde el sistema de fincas se expandió considerablemente, sobre todo a partir del último tercio del siglo XIX, cuando la agricultura comercial en todo el estado cobró un fuerte impulso.

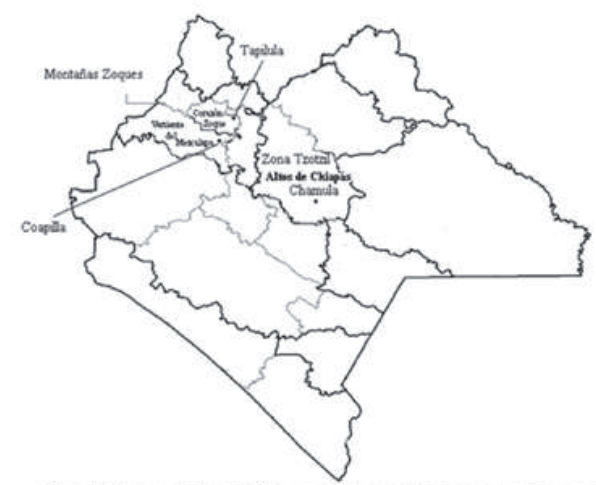

Mapa 1. Regiones Altos de Chiapas, Vertiente del Mezcalapa y Corazón Zoque 
Diversos estudiosos de la historia de Chiapas han referido ampliamente la forma compulsiva en la que se incorporó la población indígena de los Altos de Chiapas al trabajo de las fincas cafetaleras del Soconusco y de la zona de Motozintla, a finales del siglo XIX. Se ha dicho que el enganche, que consistía en el pago adelantado del salario a los trabajadores indígenas, se convirtió en una estrategia de coerción eficaz para garantizar el suministro de mano de obra a las plantaciones de café del Soconusco, región de la que dependió prácticamente la economía chiapaneca en el último tercio del siglo XIX y las primeras décadas del siglo XX.

De todos los pueblos de los Altos, el de Chamula era el que concentraba el mayor número de habitantes de toda la región, y por tanto el que más trabajadores aportaba a las fincas de café. Incluso, en la década de 1890, ante la resistencia que los propios indígenas comenzaron a presentar para trabajar en las fincas cafetaleras del Soconusco, dadas las difíciles condiciones de trabajo y los abusos que cometían los enganchadores, el gobierno del estado creó el Partido de Chamula, una jurisdicción administrativa independiente, cuyo jefe político se encargaba de vigilar muy de cerca el suministro de la mano de obra para las fincas del Soconusco y de Motozintla (Rus, 2001). Para buena parte de los habitantes de Chamula, la creación de este partido implicó mayores presiones sobre su vida social y económica, que se sumó a las que de por sí enfrentaban por la pobreza de sus tierras y la presión que sobre ellas ejercían importantes finqueros de la región. En este contexto, para muchas familias tzotziles de Chamula, y de otros pueblos de los Altos, la migración a regiones distintas del Soconusco y Motozintla representaba la posibilidad de liberarse del sistema de enganche y acceder a mejores condiciones de vida.

\section{Los migrantes tzotziles en Tapilula}

Tapilula se localiza al noroeste de Chiapas, en la región conocida como las Montañas Zoques. Esta región limita al sur y al oeste con el río Grijalva o río Grande de Chiapa, al norte con las Llanuras del Golfo y al este con los valles que convergen en las zonas de Simojovel y Huitiupán. A diferencia de otras regiones de Chiapas, las Montañas Zoques se caracterizan por la gran diversidad de paisajes que la integran: desde tierras cálidas, templadas y frías propicias para la agricultura, hasta zonas secas y poco fértiles; áreas de relieve muy abrupto, en contraste con valles extensos, situados sobre todo en la zona limítrofe con las llanuras del Golfo.

Más específicamente, el pueblo de Tapilula está ubicado en una subregión de las montañas zoques conocida como el Corazón Zoque, que se localiza al noreste de la vertiente del Mezcalapa, en un conjunto montañoso de difícil acceso debido a sus empinadas cañadas formadas por las abundantes lluvias que caen en la zona. A diferencia de la mayor parte de los pueblos del Corazón Zoque, que en general poseen tierras muy pobres, buena parte de las tierras pertenecientes a Tapilula son fértiles y presentan condiciones aptas para el cultivo de distintos productos.

Gracias a la fertilidad de sus tierras y al auge de la agricultura comercial que se presentó en todo el estado durante el último tercio del siglo XIX y la primera década del siglo XX, el número de fincas en el pueblo de Tapilula creció de manera considerable durante esos años: de cien fincas y cincuenta ranchos que se localizaban en los distintos pueblos del departamento del Mezcalapa, ${ }^{1}$ entre ellos Tapilula, en 1896, aumentaron a diecinuevefincas, 310 ranchos y 349 medianas y pequeñas propiedades rurales en 1909. De éstas, 13\% correspondía a las fincas y ranchos de Tapilula. La expansión del sistema de fincas en todo el departamento del Mezcalapa dio como resultado el aumento del número de peones o mozos arraigados en las fincas: de unos cuantos en la década de 1870, se incrementaron a 747 en $1898 ;{ }^{2}$ a 1,471 en 1909; ${ }^{3}$ y a 3,789 en $1910 .{ }^{4}$

La mayor parte de las fincas que se establecieron en Tapilula, a finales del siglo XIX, producían café y caña de azúcar, principalmente. La mayoría de ellas, pequeñas y medianas propiedades de entre diez y mil hectáreas. En ellas laboraban, en calidad de mozos o peones endeudados, $18 \%$ de los campesinos, el resto de los campesinos 
Cuadro 1. Fincas rústicas en pueblos de los departamentos de Mezcalapa, 1908

\begin{tabular}{|r|c|r|r|r|}
\hline Departamentos & Municipios & $\begin{array}{c}\text { Número de } \\
\text { fincas }\end{array}$ & $\begin{array}{c}\text { Valor de la } \\
\text { propiedad } \\
\text { rústica }\end{array}$ & $\begin{array}{c}\text { Valor promedio } \\
\text { de la propiedad } \\
\text { rústica }\end{array}$ \\
\hline \hline Mezcalapa & Coapilla & 23 & 15,811 & 689 \\
\hline \hline & Copainalá & 175 & 146,940 & 839 \\
\hline \hline & Chicoasén & 21 & 16,110 & 767 \\
\hline \hline & Ishuatán & 22 & 20,620 & 937 \\
\hline \hline & Magdalena & 137 & 55,945 & 408 \\
\hline \hline & Ocotepec & 4 & 10,800 & 2,700 \\
\hline \hline & Pantepec & 2 & 7,800 & 3,900 \\
\hline \hline & Quechula & 79 & 222,056 & 2,810 \\
\hline \hline & San Bartolomé & 13 & 22,234 & 1,710 \\
\hline \hline & Solistahuacán & & & 454 \\
\hline & Tapilula & 90 & 40,890 & 91 \\
\hline & Tecpatán & 112 & 102,458 & \\
\hline
\end{tabular}

Fuente: AHCH, Censo agrícola 1909, Valor fiscal de la propiedad raízen el estado, Secretaría General de Gobierno, Sección de Estadística, 1909.

\section{Gráfica 1. Porcentaje de población viviendo en hacienda y rancho en Tapilula}

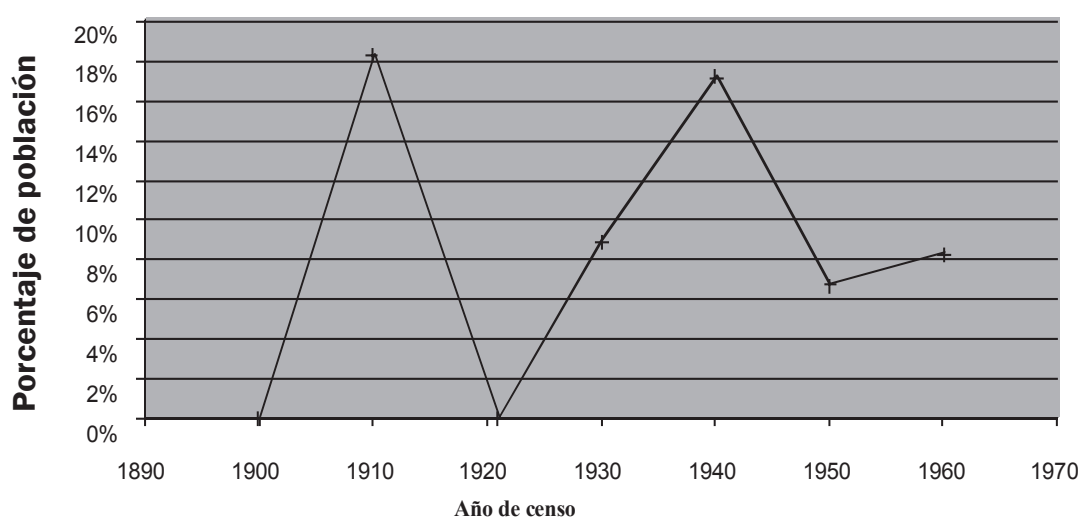

Fuentes: III Censo de población de los Estados Unidos Mexicanos, 1910, Secretaría de Agricultura y Fomento, Dirección General de Estadística, México, 1918. V Censo de población 1930, Estado de Chiapas, Secretaría de Economía Nacional, Dirección General de Estadística, México, 1935. VICenso de Población 1940, Chiapas, Secretaría de Economía Nacional, Dirección General de Estadística, México, 1943. VII Censo de Población 1950, Chiapas, Secretaría de Economía Nacional, Dirección General de Estadística, México, 1952. IXCenso de Población 1970, Chiapas, Secretaría de Economía Nacional, Dirección General de Estadística, México, 1971. 
de Tapilula mantenían la posesión de sus antiguas tierras de ejido, que se les habían concedido desde la época colonial, y que los gobiernos del siglo XIX conservaron y en algunos casos ampliaron. La retención de tierras por parte de la mayoría de los comuneros de Tapilula puede entenderse si se considera el lento crecimiento demográfico que presentó el municipio durante el siglo XIX y, en consecuencia, la escasa presión que la población ejerció sobre la tierra. Es por ello que las fincas que se establecieron en Tapilula durante los últimos años del siglo XIX y principios del XX se expandieron en terrenos nacionales y baldíos, y no a costa de los antiguos ejidos de los campesinos comuneros.

Cuadro 2. Tasa de crecimiento poblacional en los pueblos del Corazón Zoque (1778-1930)

\begin{tabular}{|l|c|c||}
\hline & $\mathbf{1 7 7 8 - 1 8 8 0}$ & $\mathbf{1 8 8 0 - 1 9 3 0}$ \\
\hline \hline Corazón zoque & $0.17 \%$ & $2.95 \%$ \\
\hline \hline Chapultenango & $0.40 \%$ & $2.89 \%$ \\
\hline \hline Francisco León & $-0.18 \%$ & $2.31 \%$ \\
\hline \hline Ocotepec & $0.59 \%$ & $2.67 \%$ \\
\hline \hline Pantepec & $-0.27 \%$ & $4.86 \%$ \\
\hline \hline Rayón & $0.56 \%$ & $2.48 \%$ \\
\hline \hline Tapalapa & $-0.29 \%$ & $2.56 \%$ \\
\hline \hline Tapilula & $0.72 \%$ & $3.32 \%$ \\
\hline \hline Total Chiapas & $1.07 \%$ & $1.53 \%$ \\
\hline
\end{tabular}

Fuente: cuadro elaborado por Juan Pedro Viqueira, INEGI-Gobierno del Estado de Chiapas, Anuario estadístico de Chiapas, ediciones 2001 y 2002, Aguascalientes, Aguascalientes 2002.

El único cambio que las tierras ejidales de Tapilula experimentaron en esos años fue en cuanto al régimen de propiedad. En agosto de 1892, el gobernador Emilio Rabasa promulgó un decreto que autorizaba al ejecutivo realizar la venta de los ejidos. ${ }^{5}$ El reglamento del decreto, publicado en 1893, establecía que el gobierno adjudicaría en propiedad privada, y mediante venta, los lotes de ejido a los propios usufructuarios, a precio de tarifa, mientras que a los campesinos de escasos recursos se les dotarían terrenos gratuitos. ${ }^{6}$ La aplicación de este decreto en el pueblo de Tapilula se inició en 1894, cuando el jefe de la sección de fomento del gobierno del estado y el ingeniero Virgilio Figueroa firmaron un contrato para llevar a cabo la remedida y el reparto de los ejidos en los departamentos de Mezcalapa y Pichucalco. ${ }^{7}$ En el municipio de Tapilula, la venta de los ejidos concluyó en 1912, cuando los comuneros, gracias a los ingresos que obtenían por el comercio de café, lograron reunir recursos suficientes para adquirir en propiedad privada lotes del antiguo ejido. ${ }^{8}$

Así, para principios de la década de 1910, una parte de la tierra de Tapilula estaba en manos de particulares que desde finales del siglo XIX poseían medianas y pequeñas propiedades. Otra parte la ocupaban pequeños ranchos, de entre cinco y diez hectáreas, que establecieron los campesinos comuneros después de la división y adjudicación del antiguo ejido en propiedad privada. El resto de las tierras de Tapilula constaban de terrenos nacionales que años atrás el gobierno del estado había incautado a la Compañía Americana Deslindadora de Terrenos. Una parte de esos terrenos se llamaba San Lorenzo, y precisamente ahí, desde 1916, varias familias de migrantes indígenas, originarias de San Juan Chamula, habían establecido sus cultivos. ${ }^{9}$

Como ocurría con la mayor parte de las tierras de Tapilula, los terrenos de San Lorenzo eran propicios para la agricultura, especialmente para el cultivo de café y caña de azúcar, productos que alcanzaron un alto valor comercial en esos años a raíz del auge de la agricultura comercial en todo el estado. De hecho, gracias a las ganancias que obtenían de la comercialización de café a pequeña escala, las familias migrantes estuvieron en posibilidad de enfrentar las presiones que en 1918 comenzó a ejercer sobre sus tierras Jesús Rico, un pequeño propietario que en ese año denunció los terrenos San Lorenzo con la finalidad de ensanchar su propiedad, y exigió a los indígenas el pago por su arrendamiento. Los 
indígenas, por su parte, enviaron una queja dirigida al gobernador del estado, en la que argumentaron que el terreno de San Lorenzo "aún no había sido adjudicado a Jesús Rico" y que estaban dispuestos "a pagar la renta del terreno al gobierno del estado", con sus propios ingresos, o bien adquirirlo en compra venta. ${ }^{10}$ En respuesta, las autoridades estatales no sólo autorizaron a los migrantes continuar usufructuando las tierras, sino que además les permitieron poseerlas por tiempo indefinido, sin cobro alguno y con la única condición de que las cultivaran."

El apoyo que los migrantes recibieron por parte del gobierno del estado hizo que Jesús Rico desistiera de su intención de apropiarse del terreno. Sin embargo, en 1925, cuando la reforma agraria comenzaba a surtir sus primeros efectos en Chiapas, y previendo que los indígenas solicitaran en dotación los terrenos San Lorenzo, Jesús Rico envió una queja a las autoridades estatales denunciando a los migrantes por estar invadiendo esos terrenos, que según él había comprado años atrás a la Compañía Americana Deslindadora de Terrenos. ${ }^{12}$ En esa ocasión, las autoridades agrarias dieron su apoyo al propietario, y a mediados de 1928, la Secretaría de Agricultura y Fomento ordenó a los migrantes desalojar los terrenos. No obstante, argumentando nuevamente que las tierras que ocupaban no le pertenecían a Jesús Rico, los campesinos se negaron a desalojar los terrenos. ${ }^{13}$ Finalmente, en 1930, después de varias gestiones, los indígenas lograron que las hectáreas que ocupaban fueran reconocidas como tierras nacionales ${ }^{14}$, y ese mismo año, en censo de población registró al núcleo de población tzotzil como ranchería "San Lorenzo", con un total de cuarenta y cuatro habitantes.

De esta manera, y pese a las dificultades que los migrantes enfrentaron con el finquero Jesús Rico, las condiciones que encontraron para establecerse de manera definitiva en el pueblo de Tapilula fueron favorables. Como vimos, no solamente recibieron el apoyo del gobierno estatal para usufructuar una parte de los terrenos San Lorenzo, y más tarde para poseerlos, sino que, al tratarse de terrenos propicios para el cultivo del café, lograron obtener algunos beneficios de su comercialización. Por otro lado, el hecho de que el grueso de los campesinos de Tapilula conservara sus antiguos ejidos, en forma de pequeñas propiedades, impidió que en esos años surgieran conflictos entre las familias tzotziles y los antiguos comuneros por el uso y posesión de la tierra.

\section{Los migrantes en Coapilla}

En contraste con la situación que enfrentaron los migrantes tzotziles de Tapilula, la suerte de varias familias también tztoziles que se establecieron en el municipio de Coapilla, probablemente desde finales del siglo XIX, no fue la misma. Coapilla se localiza en la zona de la vertiente del Mezcalapa, en la llamada Sierra de Tecpatán, ${ }^{15}$ un conjunto montañoso que se inicia al norte del cañón del Sumidero y que se caracteriza por sus profundos cañones y las numerosas corrientes de agua que forma el río Grijalva a su paso por las montañas. Algunas de estas corrientes dieron origen a valles bien irrigados y propicios para la agricultura, sobre todo en la parte norte, mientras que hacia el sur prevalecen tierras poco irrigadas y de baja productividad.

A diferencia de Tapilula, en su mayor parte, de las tierras de Coapilla son terrenos poco fértiles, por lo que los campesinos del lugar solamente han cultivado granos de primera necesidad, aunque en las partes más fértiles, algunos comuneros han cosechado café. La pobreza de las tierras obligaba a la mayoría de los campesinos de Coapilla a vender diversos objetos - petates y piedras de moler - que adquirían en los pueblos cercanos, o bien se empleaban en las escasas fincas que se establecieron en las mejores tierras del municipio durante los últimos años del siglo XIX.

A principios del siglo XX, las fincas másimportantes de Coapilla eran las de Dolores Matazano, de Salvador Camacho y San Antonio. En la primera, además de campesinos originarios de Coapilla, laboraban, en calidad de mozos o peones endeudados, un grupo de indígenas tzotziles, 
que seguramente habían migrado a finales del siglo XIX, cuando comenzaron a establecerse algunas fincas en el pueblo. ${ }^{16}$ La mayor parte de las fincas de Coapilla tenían una extensión de más de quinientas hectáreas, y hacia 1910 concentraban $38.9 \%$ de los habitantes del municipio, entre ellos los migrantes tzotziles. Además de maíz y frijol, las fincas del lugar producían café que los propietarios comercializaban en el mercado local y regional.

\section{Gráfica 2. Porcentaje de población viviendo en fincas y rancho de Coapilla}

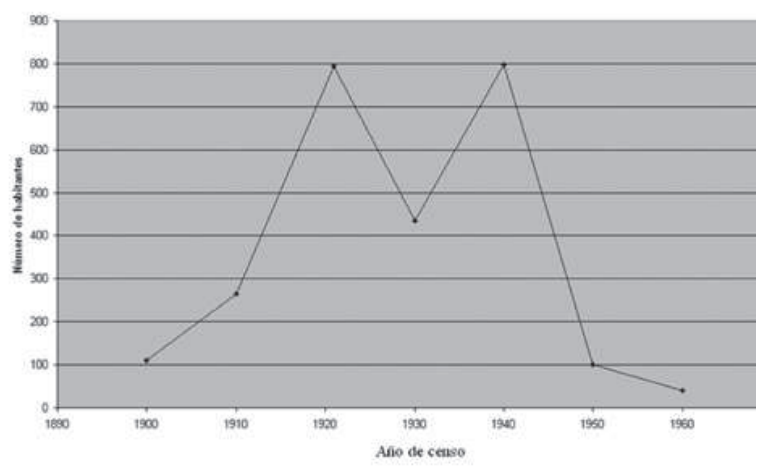

Fuentes: III Censo de población de los Estados Unidos Mexicanos, 1910, Secretaría de Agricultura y Fomento, Dirección General de Estadística, México, 1918. V Censo de población 1930, Estado de Chiapas, Secretaría de Economía Nacional, Dirección General de Estadística, México, 1935. VI Censo de Población 1940, Chiapas, Secretaría de Economía Nacional, Dirección General de Estadística, México, 1943. VII Censo de Población 1950, Chiapas, Secretaría de Economía Nacional, Dirección General de Estadística, México, 1952. IX Censo de Población 1960, Chiapas, Secretaría de Economía Nacional, Dirección General de Estadística, México, 1971.

Para esos años, al igual que en el pueblo de Tapilula, la mayor parte de los campesinos de Coapilla habían logrado retener sus antiguas tierras ejidales gracias a las leyes de 1892 que ordenaron dividir y adjudicar en propiedad privada las tierras ejidales a sus usufructuarios. En Coapilla, el fraccionamiento de los ejidos concluyó, como en el resto de los pueblos de la vertiente del Mezcalapa, en el año de 1912. Antes y después de ese año, solamente los campesinos que no comercializaban algún producto o aquellos que habían perdido sus tierras, se incorporaron al trabajo en las fincas. Por lo tanto, en las fincas del municipio, el grueso de la mano de obra fija lo constituían migrantes tzotziles, además de algunos campesinos procedentes del municipio vecino de Ocotepec.

\section{Cuadro 3. Población en ranchos y haciendas de varios pueblos de los departamentos de Mezcalapa y Pichucalco, 1910}

\begin{tabular}{||l|r|r|r|r|r||}
\hline \multicolumn{1}{|c|}{ Lugar } & $\begin{array}{c}\text { Población en } \\
\text { haciendas }\end{array}$ & $\begin{array}{c}\text { Población } \\
\text { en } \\
\text { ranchos }\end{array}$ & $\begin{array}{c}\text { Población total } \\
\text { en haciendas y } \\
\text { ranchos }\end{array}$ & $\begin{array}{c}\text { Población } \\
\text { total }\end{array}$ & $\begin{array}{c}\text { Porcentaje } \\
\text { de habitantes } \\
\text { en ranchos y } \\
\text { haciendas }\end{array}$ \\
\hline \hline Coapilla & 181 & 84 & 265 & 680 & 38.9 \\
\hline \hline Copainalá & 219 & 294 & 513 & 4,195 & 12.23 \\
\hline \hline Chicoasén & 116 & 63 & 179 & 924 & 19.37 \\
\hline \hline Magdalena & & 302 & 302 & 1,306 & 23.12 \\
\hline \hline Ocotepec & & 123 & 123 & 1,094 & 11.24 \\
\hline \hline Pantepec & 170 & 126 & 296 & 1,252 & 23.64 \\
\hline \hline Quechula & 872 & 22 & 894 & 1,237 & 72.27 \\
\hline \hline Tapalapa & 180 & 6 & 186 & 779 & 23.88 \\
\hline \hline Tapilula & 163 & 16 & 179 & 983 & 18.21 \\
\hline \hline Tecpatán & & 852 & 852 & 2,098 & 40.61 \\
\hline \hline Chapultenango & 147 & 76 & 223 & 1,246 & 17.90 \\
\hline
\end{tabular}

Fuente: Censo, División territorial del estado de Chiapas verificado en 1910, Tuxtla Gutiérrez, 1912.

En la memoria de los campesinos de Coapilla existe el recuerdo de la importancia que tuvieron las familias indígenas en el trabajo de las fincas y, en contraste, el reducido número de campesinos originarios del municipio que laboraron en ellas. Nemorio Pérez Pérez, por ejemplo, un viejo campesino de 88 años, oriundo de Coapilla, rememora cómo incluso los trabajadores indígenas que laboraban en las fincas aprendieron a hablar español gracias a la labor de algunos maestros:

El maestro del pueblo trabajaba en una finca que pertenece a Coapilla. Dolores se llamaba la finca. Otras se 
llamaban El Molino, Buenavista y San Sebastián. Ahí empezaron a hablar español, llegaron unos maestros que vinieron de quién sabe dónde. Muy poquitos de aquí fueron a trabajar a las fincas, más trabajaban en las fincas los indígenas. ${ }^{17}$

Otro campesino, Ricardo Pérez Estrada, de 87 años, también originario de Coapilla, relata la situación de los indígenas que laboraban en las fincas del pueblo, en particular los lazos que los unían con los finqueros y la dificultad que tuvieron cuando, durante los años de la Revolución, se les obligó a abandonar las propiedades:

Llegaba gente de otrolado, vinieron de Blanca Rosa, que pertenece a Ocotepec. De otros lados también vinieron, como por ejemplo, los chamulitas. Ellos vinieron hace muchos años, son indígenas, hablaban tzotzil, vinieron a trabajar. Les dieron sus terrenos; estaban en San Juanito y Llano Grande, ahí se acomodaron. Antes, tenían su dueño, su amo, el que los sostenía, y cuando los corrieron dicen que no querían salir. ${ }^{18}$

Durante los primeros años del siglo XX, las condiciones que vivieron los migrantes en las fincas de Coapilla fueron muy difíciles, pues además de percibir un salario simbólico y de cubrir jornadas extenuantes, permanecían atados a las fincas debido a las deudas a las que estaban sujetos. No obstante, como refieren los propios campesinos del lugar, los indígenas establecieron vínculos morales con los finqueros, quienes los compensaron como si compensaran las difíciles condiciones de trabajo que enfrentaban, y reforzaban así su arraigo en las fincas.

Fue a mediados de la década de 1910 cuando la situación de las familias tzotziles comenzó a modificarse. En 1914, el gobernador del estado, Agustín Castro, puso en marcha distintas reformas sociales derivadas de la revolución carrancista, entre ellas la liberación de la servidumbre por deudas, por lo que muchos finqueros fueron obligados a liberar a los trabajadores de las fincas, exone- rándolos de las deudas que habían contraído. Como en muchos otros lugares del estado, en Coapilla, los peones acasillados se resistieron a abandonar las fincas, debido a la protección y seguridad que en ellas encontraban. Nemorio Pérez recuerda, precisamente, cómo la seguridad que las fincas ofrecían a los trabajadores indígenas influyó para que éstos se resistieran a dejarlas:

Antes casi no venía gente de fuera, porque estaba muy aislado por aquí, muy pocos venían, algunos en un descuidito, aunque sufriendo en el camino, pero venían por acá, como mi papá que se vino acá cuando liberaron a los mozos, cuando la revolución. Liberaron a los mozos, los sacaron a todos, quedaron los dueños de las fincas solos, porque si no entregaban a los mozos, los mataban. El patrón correteaba a los mocitos que tenían. Había fincas que tenía cincuenta hombres, otras cien. Mi papá era hijo de de uno de ellos, ahí se crió, en el trabajo, en una finca, se llamaba Soteapa, ahorita es una colonia, se llama Javier Esquinca. Cuando aventaron a los mozos, hasta lloraban porque no sabían dónde iban a vivir con sus familias, porque estaban habituados a que el patrón les daba todo, y nunca salían de ahí, pedían y pedían, no se daban cuenta que estaba subiendo la cuenta, y no salían. Cuando salió mi papá, por acá se vino él. ${ }^{19}$

No se sabe si los migrantes tzotziles abandonaron las fincas en ese año o si permanecieron en ellas durante algún tiempo. Lo cierto es que a finales de la década de 1930, las familias indígenas recibieron en dotación terrenos localizados en las inmediaciones de la finca Dolores Matazano, una de las más grandes del pueblo. En esos años, el reparto agrario en todo el estado cobró un fuerte impulso a raíz de la política agraria que se inició con Abelardo Rodríguez y Lázaro Cárdenas. El Código Agrario de 1934, promulgado durante el gobierno de Abelardo Rodríguez, se impuso a los gobiernos estatales como el marco jurídico nacional que normaba 
el proceso del reparto agrario y en adelante, los decretos agrarios emitidos por las legislaturas estatales debieron sujetarse a la política agraria nacional.

En Chiapas, la puesta en marcha de esta nueva política agraria dio como resultado que el número de hectáreas repartidas entre 1930 y 1939 se quintuplicara con respecto a las entregadas en la década anterior (Reyes, 2002: 87). En el caso de Coapilla, desde principios de los años 1930, algunos campesinos comenzaron a presentar solicitudes de tierras, pero fue hacia finales de esa década cuando se realizaron las primeras dotaciones de ejidos en el pueblo, entre ellos la de los migrantes tzotziles. Al menos una parte de los migrantes recibieron tierras que se localizaban cerca de la finca Dolores Matazano; ahí se estableció uno de los comités agrarios encargados de ejecutar las dotaciones de tierras. ${ }^{20}$

Cuadro 4. Lista de Comités Ejecutivos Agrarios de Coapilla (1939)

\begin{tabular}{|l||c|c|}
\hline Poblado & Municipio & Distrito \\
\hline \hline Coapilla & Coapilla & Mezcalapa \\
\hline $\begin{array}{l}\text { Vicente Guerrero } \\
\text { (antes San Lorenzo) }\end{array}$ & Coapilla & Mezcalapa \\
\hline \hline Dolores Matazano & Coapilla & Mezcalapa \\
\hline \hline Morelos & Coapilla & Mezcalapa \\
\hline \hline Unión Portes Gil & Coapilla & Mezcalapa \\
\hline
\end{tabular}

Fuente: AHCH, Secretaría General de Gobierno, Documentos sin clasificar, 1939, Poblados agrarios.

Como ocurrió en otras regiones del estado, la mayoría de los primeros repartos agrarios se realizaron a partir de terrenos nacionales o baldíos, por lo que buena parte de las fincas del municipio quedaron intactas, como fue el caso de la finca Dolores Matazano, cuyos terrenos no fueron repartidos. No obstante, las tierras que las familias tzotziles recibieron como ejido se encontraban ubicadas en la parte más fértil del pueblo y en ellas pudieron cultivar, además de los granos básicos, café y caña de azúcar, productos que pudieron comercializar para obtener algunas ventajas económicas.

\section{Conclusiones}

Las familias tzotziles de San Juan Chamula que emigraron a los pueblos de Tapilula y Coapilla, a finales del siglo XIX y principios del XX, como consecuencia, entre otros factores, de las presiones que enfrentaban por el sistema del enganche, se encontraron con dos lugares relativamente poco poblados, en donde la presión sobre la tierra era escasa, y en donde las fincas demandaban mano de obra. Los migrantes tzotziles estuvieron así en posibilidad de establecerse en tierras nacionales, como en el caso de los terrenos de San Lorenzo de Tapilula, o bien se incorporaron de forma menos compulsiva al trabajo de las fincas, como ocurrió en Coapilla.

Por otro lado, los migrantes tzotziles obtuvieron ventajas de las políticas agrarias que impulsaron los gobiernos de Chiapas durante la décadas de 1930 y 1940, cuando el reparto agrario cobró fuerza en todo el estado. Como resultado de esas políticas, tanto los migrantes indígenas que se establecieron en Coapilla como los que ocuparon tierras en Tapilula recibieron dotaciones de tierras que les garantizaron por algún tiempo su sobrevivencia, pues en ambos casos se trataba de tierras fértiles, que aprovecharon para cultivar café, de cuya comercialización, por lo menos durante los años que ese grano mantuvo un precio favorable en el mercado, obtuvieron algunos beneficios.

\section{Notas}

${ }^{1}$ Archivo Histórico de Chiapas (AHCH), datos estadísticos del estado de Chiapas recopilados en el año de 1896.

${ }^{2} \mathrm{AHCH}$, Periódico oficial del estado, Tuxtla Gutiérrez, 30 de julio de 1898. 
${ }^{3}$ AHCH, Estadística agrícola de 1909, Censo agrícola 1909, Secretaría General de Gobierno, Sección de Estadística. Valor fiscal de la propiedad raíz en el estado, 1909.

${ }^{4}$ 3er. Censo de población de los Estados Unidos Mexicanos, 1910, Secretaría de Agricultura y Fomento, Dirección General de Estadística, México, 1918

${ }^{5}$ AHCH, Secretaría General de Gobierno, Sección de Fomento, tomo IX, Exp. 16-24, 1912, Decreto No. 4 que deroga la ley del 11 de agosto de 1892 en el que se autorizó al ejecutivo para la venta de los ejidos, Tuxtla Gutiérrez, 20 de septiembre de 1912.

${ }^{6} \mathrm{AHCH}$, Impresos, tomo II, Ley y reglamento para la división y reparto de ejido en el estado de Chiapas 1893, Chiapas, Imprenta del estado, 1893.

${ }^{7}$ AHCH, Secretaría General de Gobierno, Sección de Fomento, tomo III, Exp. 12, 1908, Inventario general de los expedientes y planos que se relacionan con la oficina general de ejidos en el estado, formado de los inventarios anuales de la Sección de Fomento y Obras Públicas de la Secretaría General de Gobierno correspondiente a los años de 1892 a 1907.

${ }^{8}$ AHCH, Secretaría General de Gobierno, Sección de Fomento, tomo V, Exp. No. 25, 1912, Informe del jefe político de Mezcalapa, Tuxtla Gutiérrez, 26 de diciembre de 1912.

${ }^{9} \mathrm{AHCH}$, Secretaría de Gobernación, Sección de Hacienda, tomo XIII, 1919; tomo XVIII, 1921; tomo XV, 1922.

${ }^{10} \mathrm{AHCH}$, Secretaría General de Gobierno, Sección de Fomento, tomo (ilegible), 1918, de Marcos y Sebastián Hernández y Manuel Pérez, Tapilula, l de septiembre de 1918.

"AHCH, Secretaría General de Gobierno, Sección de Fomento, tomo (ilegible), 1918, Del secretario general de gobierno a los CC. Marcos y Sebastián Hernández y Manuel Pérez, Tapilula, 6 de septiembre de 1918.

${ }^{12} \mathrm{AHCH}$, Secretaría General de Gobierno, Sección de Fomento, tomo VIII, 1925, De Jesús Rico al gobernador del estado, Tapilula, 28 de febrero de 1925.

${ }^{13}$ AHCH, Secretaría General de Gobierno, Sección de Fomento, tomo II, 1929, Del secretario de gobierno al juez mixto de Mezcalapa, Tuxtla Gutiérrez, 8 de abril de 1929.
${ }^{14}$ AHCH, Secretaría General de Gobierno, Sección de Fomento, documentos sin clasificar, 1930, De i? al secretario general de gobierno, 1930.

${ }^{15}$ Dentro de las Montañas Zoques, Juan Pedro Viqueira distingue cuatro subregiones con características geográficas comunes, pero que durante la Colonia desempeñaron funciones económicas específicas. Estas regiones son: las sierras de Tecpatán y Tapalapa o Pantepec, las estribaciones de Chapultenango y los Valles de Jitotol. Véase Cronotopología de una región rebelde. La construcción histórica de los espacios sociales en la Alcaldía Mayor de Chiapas (1520-1720), tesis doctoral, EHESS Paris, Francia, 1997.

${ }^{16}$ Cuadro sinóptico estadístico de la República Mexicana, División Territorial de la República Mexicana, Dirección General de Estadística, Estados del Centro, México, Oficina telegráfica de la Secretaría de Fomento, 1904.

${ }^{17}$ Entrevista realizada en noviembre de 2004.

${ }^{18}$ Entrevista realizada en noviembre de 2004.

${ }^{19}$ Entrevista realizada en noviembre de 2004.

${ }^{20}$ Relación de resoluciones presidenciales en Chiapas: 1940-1956, citado en María Eugenia Reyes Ramos, Conflicto Agrario en Chiapas: 1934-1964,op. cit., p. 422.

\section{Bibliografía}

Anuario estadístico del estado de Chiapas. Año de 1909, 1911, Tuxtla Gutiérrez, Tipografía del Gobierno de Chiapas.

Baumann, Friederike, 1983, "Terratenientes, campesinos y la expansión de la agricultura capitalista en Chiapas, 1896-1916", Mesoamérica, pp. 8-63.

Benjamin, Thomas, 1984, El camino a Leviatán, México, Consejo Nacional para la Cultura y las Artes, 1990.

Favre, Henri, Cambio y continuidad entre los mayas. Contribución al estudio de la situación colonial en América Latina, México, INI.

Lisbona Guillén, Miguel, 2000, En tierra zoque. Ensayos para leer una cultura, Consejo Estatal para la Cultura y las Artes, Tuxtla Gutiérrez, Chiapas. 
Pozas, Arciniega Ricardo, 1952, "El trabajo en las plantaciones de café y el cambio sociocultural del indio", en Revista Mexicana de Estudios Antropológicos, XII, pp. 31-48.

—, 1977, Chamula. Un pueblo indio de los Altos de Chiapas, 2 vols., México, INI.

Reyes Ramos, María Eugenia, 2002, Conflicto Agrario en Chiapas: 1934-1964, UAM-CONECULTA, Gobierno del estado de Chiapas, México, p. 87.

-, 1992, El reparto de tierras y la política agraria en Chiapas, 1914-1988, UNAM y CIHMECH.
Rus, Jan, 2001, "Contained revolutions: The Struggle for Control ok Highland Chiapas. 1910-1925", Mexican Studies/Estudios Mexicanos (Berkeley, Universidad de California).

Viqueira Albán, Juan Pedro, 1995, "Chiapas y sus regiones", Chiapas. Los rumbos de otra historia, México, Universidad Nacional Autónoma de México.

—, 1997, Cronotopología de una región rebelde. La construcción histórica de los espacios sociales en la Alcaldía Mayor de Chiapas (1520-1720), tesis doctoral, EHESS Paris, Francia. 